A. G. Endegnanew, K. Uhlen, G. Bergna-Diaz and J. Beerten, "Small-signal modelling of an MMC-based HVDC link interfacing large AC systems," in Proc. IEEE 17th Workshop on

Control and Modeling for Power Electronics (COMPEL) 2016, Trondheim, Norway, 27-30 Jun. 2016, 9 pages.

Digital Object Identifier: 10.1109/COMPEL.2016.7556765

URL:

http://ieeexplore.ieee.org/document/7556765/

(C) 2016 IEEE. Personal use of this material is permitted. Permission from IEEE must be obtained for all other users, including reprinting/ republishing this material for advertising or promotional purposes, creating new collective works for resale or redistribution to servers or lists, or reuse of any copyrighted components of this work in other works. 


\section{Small-signal Modelling of an MMC-based HVDC Link Interfacing Large AC Systems}

\author{
Atsede G. Endegnanew \\ Kjetil Uhlen \\ Department of Electric Power Engineering \\ NTNU \\ Trondheim, Norway \\ atsede.g.endegnanew@ntnu.no
}

\author{
Gilbert Bergna-Diaz \\ SINTEF Energy Research \\ Trondheim, Norway
}

\author{
Jef Beerten \\ Dept.of Electrical Engineering \\ KU Leuven - EnergyVille \\ Heverlee - Genk, Belgium
}

\begin{abstract}
This paper presents a comprehensive modelling approach to obtain a small-signal model of a Modular Multilevel Converter (MMC)-based High Voltage Direct Current (HVDC) link interfacing two large asynchronous ac systems. Detailed models are used to represent synchronous generators, MMC converters and their controllers. Timedomain simulations of the non-linear model are presented to test the performance the developed model of the hybrid ac/dc power system. A small-signal analysis is used to assess the stability of the overall system.
\end{abstract}

Keywords-Small Signal Stability, AC Networks, Modular Multilevel Converters, HVDC

\section{INTRODUCTION}

As HVDC links are used to interconnect ac system and operate in parallel with them, it is important to study the dynamics of the entire hybrid ac/dc system. Until now, most papers focused on either the High Voltage Direct Current (HVDC) system stability [1-4], thereby leaving out the ac system stability or included on the ac side stability but simplified the de systems to a power injection model [5]. Ref. [6, 7] have studied small signal stability of a hybrid ac/dc power system. In both papers the dc system consists of two-level Voltage Source Converters (VSC). Nonetheless, it is the Modular Multilevel Converter (MMC) that is establishing itself as the most promising VSC for HVDC transmission schemes $[8,9]$. The MMC topology provides significant advantages for HVDC applications compared to the two-level predecessor, such as lower losses, modularity, scalability and low harmonic content in the output ac voltage. However, the modelling and control of MMCs present additional challenges compared to two-level VSCs, since the internal dynamics of this converter contain additional state and control variables, with multiple frequency components in steady state operation. As opposed, to the two-level converter [10], small-signal models of the MMC which are useful for applying traditional eigenvaluebased techniques for stability studies, have still not reached a state of maturity, albeit the recent appearance of several promising approaches, such as $[3,11,12]$ to name a few. However, these small-signal models do not include in full detail the ac networks to which the MMCs are connected.
Instead, the ac system is represented by an equivalent Thevenin source behind a Thevenin impedance.

This paper intends to contribute to the emerging state of the art on small signal modelling of hybrid ac/dc power systems by developing a small-signal model an MMC based HVDC link interfacing ac power systems. The differential and algebraic equations (DAE) for all the dynamic components in the ac grid, MMC control and the dc cable are presented in detail. The developed model was linearized and was used to study small signal stability and dynamic interaction in the hybrid system.

\section{HYBrID AC/DC Test POWER SYSTEM}

In this paper, a combined dynamic model for a hybrid ac/dc power system is developed for small signal stability studies. A power system with two asynchronous multimachine ac grids and an MMC-based HVDC link is used as a test system for the dynamic model development, shown in Fig. 1. Grid 1 has four 900 MVA generators connected by a long transmission line. It is based on the two-area system in [13]. Grid B is a 9 bus system with three-generator and three loads [14]. All machines in the test system are synchronous generators with automatic voltage regulators and governor controllers. Gen 1 in Grid A is also equipped with a power system stabilizer (PSS) for damping power system oscillations.

Both of the MMC converters illustrated in Fig. 1 are modeled using the simplified approach presented in [3], based on zero-sequence representation of internal energy and current, allowing for constant state and control variables in steady-state. This approach is accurate under the assumptions given in [3] and recalled in section IV, and allows for obtaining a linearized version of the model intended for small-signal studies. However, such assumptions impose the additional requirement for an internal stabilizing energy control of the converter, also described in section IV. 


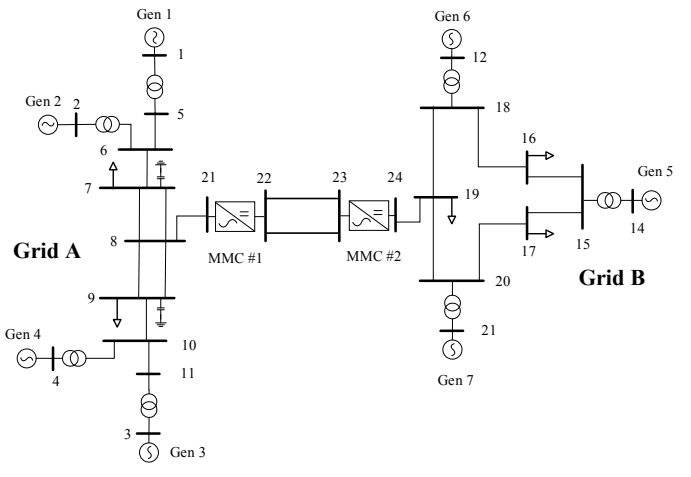

Fig. 1. Hybrid ac/dc test system

In power system dynamic studies, it is crucial to use models that properly capture the dynamic behavior of power system components such as generators, converters and their controllers. Differential and algebraic equations (DAE) typically form the basis of models of the power system components that are used in dynamic studies. The following sections present the DAE equations for the dynamic models of synchronous generators and MMC converters, and their controllers. Furthermore, the dynamic model for the de link cable used in the test system is presented in section $\mathrm{V}$.

\section{AC GRID COMPONENTS}

\section{A. Generators}

A $6^{\text {th }}$ order model is used for the synchronous generators in the test system to represent machines with damper windings on both $\mathrm{d}$ and $\mathrm{q}$ rotor axes. The differential equations for each synchronous generator $i$ are [15]:

$$
\begin{aligned}
& \dot{\delta}_{i}=\omega_{0}\left(\omega_{i}-\omega_{s}\right)=\omega_{0} \Delta \omega_{i} \\
& \Delta \omega_{i}=\frac{1}{2 H_{i}}\left(P_{m i}-P_{e i}-D_{i} \Delta \omega_{i}\right) \\
& \dot{E_{q i}^{\prime}}=\frac{1}{T_{d 0 i}^{\prime}}\left(E_{f d i}-E_{q i}^{\prime}+I_{d i}\left(x_{d i}-x_{d i}^{\prime}\right)\right) \\
& \dot{E_{d i}^{\prime}}=\frac{1}{T_{q 0 i}^{\prime}}\left(-E_{d i}^{\prime}-I_{d i}\left(x_{q i}-x_{q i}^{\prime}\right)\right) \\
& \dot{E_{q i}^{\prime \prime}}=\frac{1}{T_{d 0 i}^{\prime \prime}}\left(E_{q i}^{\prime}-E_{q i}^{\prime \prime}+I_{d i}\left(x_{d i}^{\prime}-x_{d i}^{\prime \prime}\right)\right) \\
& \dot{E_{d i}^{\prime \prime}}=\frac{1}{T_{q 0 i}^{\prime \prime}}\left(E_{d i}^{\prime}-E_{d i}^{\prime \prime}-I_{q i}\left(x_{q i}^{\prime}-x_{q i}^{\prime \prime}\right)\right)
\end{aligned}
$$

where $\delta_{i}$ is the angle, in radians, between each machine's rotor q-axis and the reference machine's rotor q-axis, $\omega_{i}$ is rotor speed, $\Delta \omega_{i}$ is the speed deviation, $E_{i}$ ' and $E_{i}{ }^{\prime}$ ' are transient and sub-transient voltages while $x, x^{\prime}$ and $x$ " are synchronous, transient and sub-transient reactances, respectively. $H_{i}$ is the inertia constant in seconds, and $T_{0}{ }^{\prime}$ and $T_{0}{ }^{\prime \prime}$ are the open-circuit transient and sub-transient time constants, respectively. $\omega_{0}$ is the rotor base angular speed in $\mathrm{rad} / \mathrm{s}$ and $\omega_{s}$ is the synchronous speed, which is the speed of the reference machine.
The subscripts $d$ and $q$ represent $\mathrm{d}$ - and q-axis components of each variable in the individual synchronous machine's rotor d-q axis: where d-axis is aligned with the magnetic axis of the field windings and q-axis lags $\mathrm{d}$-axis by 90 electrical degrees.

Field excitation voltages $E_{f d i}$ and mechanical power $P_{m i}$ are outputs of excitation and governor system controllers, respectively. If a generator does not have such controllers, then a constant value is used in the modelling. The air gap electrical power, $P_{e i}$, is calculated from (2) and links the differential generator equations with the algebraic ac network through generator currents $I_{d i}$ and $I_{q i}$.

$$
P_{e i}=E_{d i}^{\prime \prime} I_{d i}+E_{q i}^{\prime \prime} I_{q i}-\left(x_{d i}^{\prime \prime}-x_{q i}^{\prime \prime}\right) I_{d i} I_{q i}
$$

\section{B. Generator controllers}

This subsection presents dynamic models for the excitation and governor system controllers used in the test system.

\section{1) Automactic Voltage Regulator (AVR)}

A simplified excitation system model (SEXS) is used with all generators in the test system. The governing equations for the AVR model are:

$$
\begin{aligned}
& \dot{E_{f d i}}=\frac{1}{T_{E i}}\left(K_{i} x_{A V R i}\left(1-\frac{T_{A i}}{T_{B i}}\right)+\frac{K_{i} T_{A i}}{T_{B i}}\left(V_{r e f i}-V_{t i}+V_{P S S i}\right)-E_{f d i}\right) \\
& \dot{x_{A V R i}}=\frac{1}{T_{B i}}\left(V_{r e f i}-V_{t i}+V_{P S S i}-x_{A V R i}\right)
\end{aligned}
$$

where $T_{A}$ and $T_{B}$ are lead-lag controller time constants, $K$ is the controller gain parameter and $T_{E}$ is the exciter time constant $E_{f d}$ is field voltage and $x_{A V R}$ an additional state variable associated with the lead-lag controller. The regulator takes in the reference voltage $\left(V_{r e f}\right)$ as an input, as well as the generator's terminal voltage $\left(V_{t}\right)$ and $V_{P S S}$, which is the output signal of the PSS.

\section{2) Power system stabilizer}

A power system stabilizer improves power system dynamic performance by damping system oscillations. The controller used here is a STAB1 type of controller and uses rotor speed deviation as an input. It has a washout filter and cascaded lead-lag controllers as shown in Fig. 2.

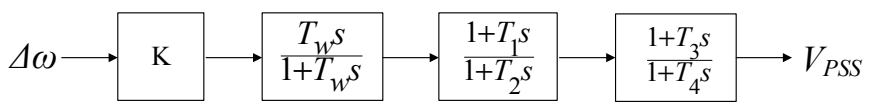

Fig. 2. STAB1 type power system stabilizer

The DAE equations for the PSS are: 


$$
\begin{aligned}
& V_{P S S i}=\frac{T_{1 i} T_{3 i}}{T_{w i} T_{2 i} T_{4 i}}\left(K_{P S S i} \Delta \omega_{i}-x_{P S S 3 i}\right)-\left(\frac{T_{1 i}-T_{2 i}}{T_{2 i} T_{4 i}}\right) T_{3 i} x_{P S S 2 i}-\frac{T_{3 i}-T_{4 i}}{T_{4 i}} x_{P S S 3 i} \\
& \dot{x_{P S S 1 i}}=\frac{1}{T_{w i}}\left(K_{P S S i} \Delta \omega_{i}-x_{P S S 1 i}\right) \\
& \dot{x_{P S S 2 i}}=\frac{1}{T_{w i} T_{2 i}}\left(K_{P S S i} \Delta \omega_{i}-x_{P S S 1 i}\right)-\frac{1}{T_{2 i}} x_{P S S 2 i} \\
& \dot{x_{P S S 3 i}}=\frac{T_{1 i}}{T_{w i} T_{2 i} T_{4 i}}\left(K_{P S S i} \Delta \omega_{i}-x_{P S S 3 i}\right)-\left(\frac{T_{1 i}-T_{2 i}}{T_{2 i} T_{4 i}}\right) x_{P S S 2 i}-\frac{1}{T_{4}} x_{P S S 3 i}
\end{aligned}
$$

\section{3) Governor}

TGOV1 is a model for a steam turbine governor. The DAE for TGOV1 type of turbine and governor model is:

$$
\begin{aligned}
& x_{G O V 1 i}^{\cdot}=\frac{1}{T_{1 i}}\left(\frac{1}{R_{i}}\left(\omega_{i}-\omega_{\text {refi }}\right)-x_{G O V 1 i}\right) \\
& \cdot \cdot \overrightarrow{G O V 2 i} \frac{1}{T_{3 i}}\left(x_{G O V 1 i}-x_{G O V 2 i}\right) \\
& P_{m}=x_{G O V 1 i}+\left(x_{G O V 1 i}-x_{G O V 2 i}\right)\left(\frac{T_{2 i}-T_{3 i}}{T_{3 i}}\right)-D_{t i} \omega_{i}
\end{aligned}
$$

where $T_{1}$ is governor time constant, $T_{2}$ and $T_{3}$ are heater time constants, $\omega$ and $\omega_{\text {ref }}$ are rotor speed and speed reference, respectively. $D_{t}$ is the turbine damping constant, $R$ is the frequency droop parameter of the turbine.

\section{MOdular Multi-LEVEl VSC CONVERTER AND CONTROLLER}

The MMC terminal has been modelled as presented in [3], and used for small-signal analysis of a two-terminal link in [2] and is briefly discussed in this section.

\section{A. Physical system and inner current control loop}

The accuracy of the simplified state-space modeling approach of [3], which is derived from by the well-known average model described in [16] and [17], is strongly dependent on the assumption of what has been referred to as Compensated Modulation (CM). This implies that the control system includes an on-line compensation for the variations in the sum of the arm capacitor voltages when calculating the insertion indexes. More precisely, the upper and lower arm insertion indexes of the converter $n_{u}$ and $n_{l}$ for each phase $k$ are calculated by dividing the output of the acgrid and internal circulating current controllers $e_{v}{ }^{*}$ and $u_{c}{ }^{*}$ by the aggregated voltage, $v_{c u}{ }^{\Sigma}$ or $v_{c l}{ }^{\Sigma}$, in the corresponding arm as indicated by equation (6).

$$
n_{u, k}=\frac{-e_{v, k}^{*}+u_{c, k}^{*}}{v_{c u, k}^{\Sigma}}, \quad n_{l, k}=\frac{e_{v, k}^{*}+u_{c, k}^{*}}{v_{c l, k}^{\Sigma}} ; \quad \text { for } \quad k \in\{a, b, c\}
$$

If this assumption is used, the voltages driving the acside currents and the circulating currents $e_{v}$ and $u_{c}$ become approximately equal to the output of their respective controllers, as indicated by (7).

$$
\begin{aligned}
& e_{v}^{a b c}=e_{v}^{*, a b c} \rightarrow e_{v}^{d q}=e_{v}^{*, d q} \\
& u_{c}^{a b c}=u_{c}^{*, a b c} \rightarrow u_{c}^{d q z}=u_{c}^{*, d q z}
\end{aligned}
$$

Additional consequences of the CM assumption includes the introduction of a significant degree of decoupling between the zero-sequence dynamics of the energy-sum $w_{\sum, z}$ from the individual energy difference oscillations $w_{\Delta}$. Furthermore, it is possible to neglect the effect of the $d q$ components of $u_{c}{ }^{*}$ in the energy-sum zero-sequence dynamics, as they are significantly smaller than the zerosequence component $u_{c z}$ [3]. From these assumptions, a simplified representation of the MMC can be obtained by modelling only the zero-sequence dynamics of the energysum $w_{\sum z}$, and the circulating current $i_{c z}$, which are inherently time-invariant in steady state, and therefore suited for linearization and traditional eigenvalue-based stability analysis.

Thus, the energy difference and the other frequency components of the circulating current can be neglected while still obtaining a model that is accurately representing an $\mathrm{MMC}$, as seen from its ac- and dc-terminals. The MMC simplified zero-sequence model is represented by (8).

$$
\begin{aligned}
& \dot{w}_{\Sigma z} \approx\left[-1 / 2\left(e_{v d}^{*} i_{v d}+e_{v q}^{*} i_{v q}\right)+2 u_{c z}^{*} i_{c z}\right] \frac{\omega_{b}}{4 c_{e q}} \\
& \dot{i}_{c z}=-\frac{\omega_{b} r_{a}}{l_{a}} i_{c z}-\frac{\omega_{b}}{l_{a}} u_{c z}^{*}+\frac{\omega_{b}}{l_{a}} v_{d c} \omega_{b}
\end{aligned}
$$

In (8), $c_{e q}$ is the total equivalent capacitance per arm of the MMC (i.e. $C_{S M} / N$ with $C_{S M}$ the submodule capacitance), $r_{a}$ and $l_{a}$ are the arm resistance and inductance of the MMC, all represented in the ac-side per unit system. All voltage and current variables in (8) are represented in the ac-side per unit system except $v_{d c}$, which is represented in the dc-side per unit system. Finally $w_{\sum z}$ is represented in the per unit system by means of the MMC energy base, defined as $W b=$ $\left(C_{S M} / N\right) *\left(V_{b, d c}\right)^{2}$.

\section{B. MMC internal dynamics control loops}

An overview of the control system is depicted in Fig. 3. The control of the zero-sequence energy sum $w_{\sum z}$ and the zero-sequence circulating current $i_{c z}$ are explicitly represented in the model. Thus, an outer loop PI controller for $w_{\sum z}$ as defined in (9) is providing the current reference for an inner loop PI controller for $i_{c z}$.

$$
\begin{aligned}
& i_{c z}^{*}=k_{p \Sigma}\left(w_{\Sigma}^{*}-w_{\Sigma}\right)+k_{i \Sigma} \kappa \\
& \dot{\kappa}=w_{\Sigma}^{*}-w_{\Sigma}
\end{aligned}
$$

In turn, the inner control loop regulates $i_{c z}$ to its reference by providing the reference value $u_{c z}{ }^{*}$, as given in (10).

$$
\begin{aligned}
& u_{c z}^{*}=-k_{p c}\left(i_{c z}^{*}-i_{c z}\right)-k_{i c} \xi \\
& \dot{\xi}=i_{c z}^{*}-i_{c z}
\end{aligned}
$$

In (9)-(10) with $k_{p \sum}, k_{p c}, k_{i \Sigma}$ and $k_{i c}$ are the proportional and integral gain coefficients of both controllers, whereas $\kappa$ 
and $\xi$ are the states created by the integral part of the PI regulator.

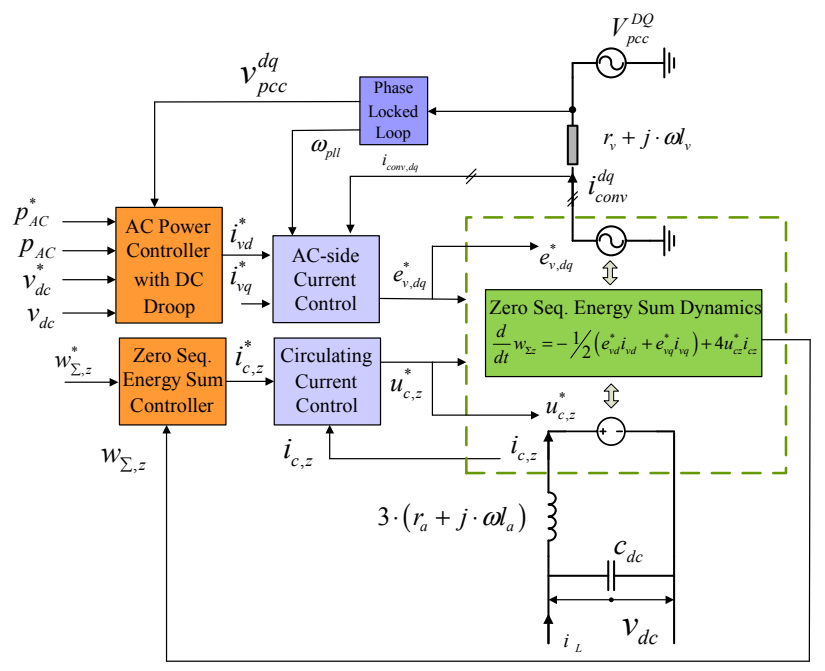

Fig. 3. Overview of configuration and control system for a single-terminal MMC

\section{AC side}

The ac-grid current dynamics can be expressed as in (11), where $\omega_{g}$ is the grid frequency in per unit while $r_{v}$ and $l_{v}$ are the equivalent resistance and inductance defined as in (12), where $r_{f}$ and $l_{f}$ are the output filter resistance and inductance.

$$
\begin{gathered}
\dot{i}_{c o n v, d}=\frac{\omega_{b}}{l_{v}}\left(e_{v, d}^{*}-v_{p c c, d}-r_{v} i_{c o n v, d}+\omega_{g} l_{v} i_{c o n v, q}\right) \\
\dot{i}_{c o n v, q}=\frac{\omega_{b}}{l_{v}}\left(e_{v, q}^{*}-v_{p c c, q}-r_{v} i_{c o n v, q}-\omega_{g} l_{v} i_{c o n v, d}\right) \\
r_{v}=\frac{r_{a}}{2}+r_{f} ; \quad l_{v}=\frac{l_{a}}{2}+l_{f}
\end{gathered}
$$

\section{AC-grid current controllers}

A phase locked loop (PLL) is used to find the angle the point of common couple (PCC) voltage vector. The rotating $\mathrm{d}-\mathrm{q}$ reference frame used in the converter control has its $\mathrm{d}-$ axis aligned with PPC voltage vector. This definition of $d-q$ axis allows a decoupled control of active and reactive power. With $v_{p c, q}=0$, active and reactive power equations become only proportional to $\mathrm{d}$ and $\mathrm{q}$ axis currents, respectively. Thus, the active and reactive power definitions at the grid connection point are given as in (13).

$$
\begin{aligned}
& p_{a c}=v_{p c c, d} i_{c o n v, d} \\
& q_{a c}=-v_{p c c, q} i_{c o n v, q}
\end{aligned}
$$

For the ac-side control, a typical implementation based on cascaded control with inner and outer control loops is used, briefly described in the following lines.

\section{1) Outer controller}

The outer controller regulates active power or dc voltage depending on the HVDC terminal, as well as the reactive power in respectively the $\mathrm{d}$ and $\mathrm{q}$ axes. The outer powerregulating control loop is given in (14), where $\rho_{d}$ and $\rho_{q}$ are the states created by the integral part of the PI regulator. In addition, $k_{p, p a c}$ and $k_{i, p a c}$ are the proportional and integral tuning parameters of the controller.

$$
\begin{aligned}
& i_{c o n v, d}^{*}=k_{p, p a c}\left(p_{a c}^{*}-p_{a c}\right)+k_{i, p a c} \rho_{d} \\
& \dot{\rho}_{d}=p_{a c}^{*}-p_{a c} \\
& i_{c o n v, q}^{*}=k_{p, p a c}\left(q_{a c}^{*}-q_{a c}\right)+k_{i, p a c} \rho_{q} \\
& \dot{\rho}_{q}=q_{a c}^{*}-q_{a c}
\end{aligned}
$$

Note that for the dc-voltage controlling station, the first two equations in (14) are replaced by (15)

$$
\begin{aligned}
& i_{c o n v, d}^{*}=-k_{p, v d c}\left(v_{d c}^{*}-v_{d c}\right)-k_{i, v d c} \rho_{v d c} \\
& \dot{\rho}_{v d c}=v_{d c}^{*}-v_{d c}
\end{aligned}
$$

\section{2) Inner controller}

The inner controller regulates the current using standard PI controllers in a Synchronously Rotating d-q Reference Frame (SRRF) including decoupling feedforward terms, as indicated by (16). $\gamma_{d}$ and $\gamma_{q}$ are the states created by the integral part of the ac-grid current inner control loop whereas $k_{p, v}$ and $k_{i, v}$ are the PI proportional and integral parameters.

$$
\begin{aligned}
& e_{v, d}^{*}=k_{p, v}\left(i_{c o n v, d}^{*}-i_{c o n v, d}\right)+k_{i, v} \gamma_{d}-l_{v} \omega_{p l l} i_{c o n v, q}+v_{p c c, d} \\
& \dot{\gamma}_{d}=i_{c o n v, d}^{*}-i_{c o n v, d} \\
& e_{v, q}^{*}=k_{p, v}\left(i_{c o n v, q}^{*}-i_{c o n v, q}\right)+k_{i, v} \gamma_{q}+l_{v} \omega_{p l l} i_{c o n v, d}+v_{p c c, q} \\
& \dot{\gamma}_{q}=i_{c o n v, q}^{*}-i_{c o n v, q} \\
& \text { 3) DC side }
\end{aligned}
$$

The dc voltage dynamics of the cable capacitance connected in between the dc terminals of the MMC is given by (17), where $c_{d c}$ is the border capacitance of the cable model that will be discussed in section V. Furthermore, $i_{L}$ is the cable current, as defined in Fig. 3. Finally, the zerosequence of the circulating current is being multiplied by 4 instead of 3 since it is referred to the ac-side per unit system.

$$
\dot{v}_{d c}=\frac{\omega_{b}}{c_{d c}}\left(i_{L}-4 i_{c, z}\right)
$$

\section{DC LINK CABLE}

In order to accurately represent the frequency dependency of the dc cable's series resistance $r$ and inductance $l$ per unit length, a cascaded pi-section model with multiple parallel RL-branches is used, as proposed in [18]. As a compromise between model complexity and accuracy, five pi-sections and five-parallel branches are included. 

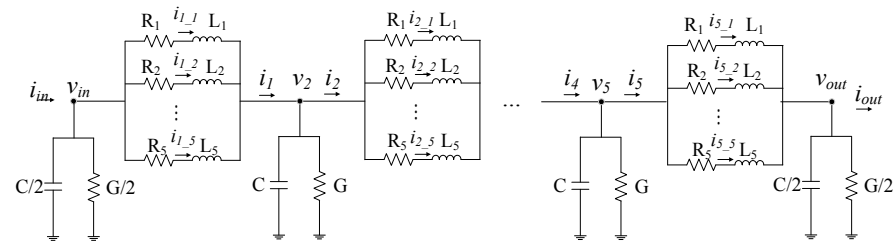

Fig. 4. Multiple pi section with multiple parallel branches cable model

Similar to traditional pi-section models, the voltage and current dynamics can, be easily written in terms of first-order differential equations, and allows straightforwardly using these equations for the small-signal studies.

Contrary to traditional pi-sections, the current in the series element of each pi-section is split amongst different parallel branches, each with their own RL dynamics, which allows to accurately account for the frequency-dependence of the series impedance. Following Kirchhoff's law, the current in the series element is equal to the sum of the currents in the parallel branches. The currents flowing in parallel branches $\left(i_{1} 1_{1}, i_{1} 2, \ldots i_{1} 5\right)$ have dynamics governed according to:

$$
\dot{i}_{1_{-} 1}=\frac{1}{L_{1}}\left(v_{1}-v_{2}-R_{1} i_{1 \_1}\right)
$$

The voltage dynamics at the beginning and end of the cable are governed by:

$$
\begin{aligned}
& \dot{v}_{\text {in }}=\frac{2}{C}\left(i_{\text {in }}-i_{1}-\frac{G}{2} v_{\text {in }}\right) \\
& \dot{v}_{\text {out }}=\frac{2}{C}\left(i_{5}-i_{\text {out }}-\frac{G}{2} v_{\text {out }}\right)
\end{aligned}
$$

whereas the voltage dynamics in the middle of the cable $\left(v_{2}, v_{3}, v_{4}, v_{5}\right)$ are governed by the following first-order differential equation.

$$
\dot{v}_{2}=\frac{1}{C}\left(i_{1}-i_{2}-G v_{2}\right)
$$

\section{COMPLETE HyBrid AC/DC POWER SySTEM}

\section{A. Reference frames}

The synchronous generators and converter control systems models use different types of synchronously rotating $\mathrm{d}-\mathrm{q}$ rotating reference frames. On the one hand, the $\mathrm{d}-\mathrm{q}$ rotating frame used for synchronous generator modeling has its d-axis aligned with the magnetic axis of the rotor field windings and leads the q-axis by 90 electrical degrees. On the other hand, the d-q reference system used for converter control modelling has its d-axis aligned with the voltage vector at the point of common coupling, and the d-axis lags the q-axis by 90 electrical degrees. A common global reference frame is necessary to connect the various components modelled in their own d-q reference frames and to solve the algebraic load flow equations. This global reference frame is usually the $\mathrm{d}-\mathrm{q}$ rotor axis of one of the machines in the ac system, called the reference machine.
Before solving the network equations, currents and voltages at each generator terminal are rotated by their rotor angle $\delta_{i}$, while the converter terminal currents and voltages are rotated by PCC voltage angle $\theta$, which is measured by a PLL. Fig. 5 shows d-q rotating reference frame for converter control, synchronous generator modelling together with their rotational shift compared to the global reference frame.

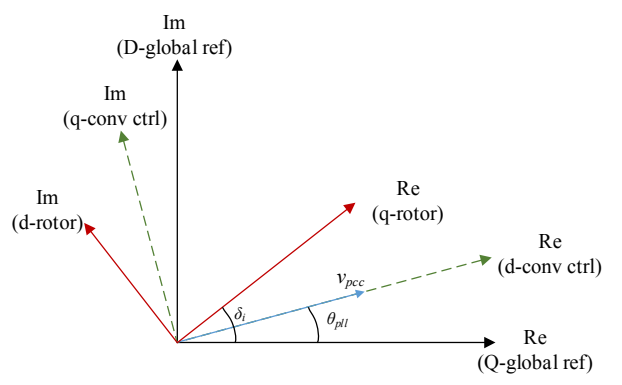

Fig. 5. Rotating reference frames

Transformation between local and global references frames is done using (21) and (22).

$$
\begin{gathered}
Q_{\text {global }}+j D_{\text {global }}=\left(q_{\text {rotor }}+j d_{\text {rotor }}\right) e^{j \delta} \\
Q_{\text {global }}+j D_{\text {global }}=\left(d_{\text {conv }}+j q_{\text {conv }}\right) e^{j \theta_{\text {pll }}}
\end{gathered}
$$

It should be noted that for the rotor $\mathrm{d}-\mathrm{q}$ system, $\mathrm{q}$ is the on real axis, while for converter $\mathrm{d}-\mathrm{q}$ frame, $\mathrm{d}$ is on the real axis.

\section{B. Phase Locked Loop (PLL)}

The PLL estimates the PCC voltage phase angle, which is the direction of the d-axis of the converter control system. This means that at steady state the PCC voltage has only a d axis component in the converter $\mathrm{d}-\mathrm{q}$ frame since the PI controller is regulating the q-axis component $\left(v_{p c c, q}\right)$ to zero. The PCC voltage $v_{p c c, q}$ calculated from (22) is fed into the PI controller as shown in Fig. 6.

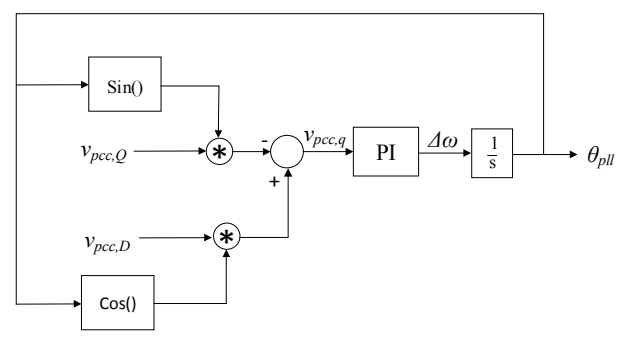

Fig. 6. PLL block diagram

The PLL is modelled by the following differential equations:

$$
\begin{aligned}
& \dot{\theta_{p l l}}=k_{p, p l l}\left(-\sin \theta_{p l l} * v_{p c c, \mathrm{Q}}+\cos \theta_{p l l} * v_{p c c, \mathrm{D}}\right)+x_{p l l} \\
& \dot{x_{p l l}}=k_{i, p l l}\left(-\sin \theta_{p l l} * v_{p c c, \mathrm{Q}}+\cos \theta_{p l l} * v_{p c c, \mathrm{D}}\right)
\end{aligned}
$$


where $k_{p, p l l}$ and $k_{i, p l l}$ are the proportional and integral parameters of the PI controller, respectively, $v_{p c c, Q}+\mathrm{j} v_{p c c, D}$ is the PCC voltage vector in the global reference frame, $\theta_{p l l}$ is the PCC voltage vector angle, and $x_{p l l}$ is a state variable associated with the integrator in the PI controller of the PLL.

\section{AC Transmission Network Equations}

Generators and converters are modelled as voltage sources behind appropriate impedances. In the network equations, generators and converters are represented by a Norton equivalent current source in parallel with their respective impedances. Fig. 7 and (24) present the equivalent circuit and corresponding equation for the generators and converters representation in network equations.

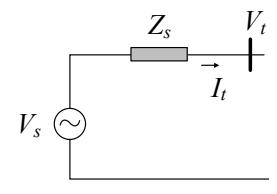

(a)

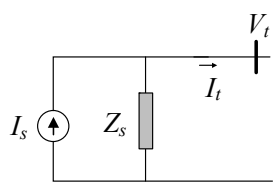

(b)
Fig. 7. Equivalent circuit for generators and converters in netowrk modelling

$$
I_{s}=\frac{V_{s}}{Z_{s}}=\frac{V_{t}}{Z_{s}}+I_{t}
$$

For a $6^{\text {th }}$ order model of the synchronous generator presented in (1), $V_{s}$ is subtransient voltage ( $\left.E^{\prime \prime}\right)$ and $Z_{s}$ is $r_{a}+j x_{d}^{\prime \prime}$, where $r_{a}$ is stator resistance. For the MMC converter model, $V_{s}$ is the converter voltage driving the ac grid current $\left(e^{*}\right)$ while $Z_{s}$ is MMC filter impedance plus half of the MMC arm inductance $\left(r_{v}+j \omega_{g} l_{v}\right)$. The admittances $1 / Z_{s}$ at generator and converter buses are augmented to the nodal admittance matrix forming $\mathbf{Y}_{\text {Aug }}$ and as a result, the overall network equations become:

$$
\mathbf{I}=\mathbf{Y}_{A u g} \mathbf{V}
$$

where $\mathbf{I}$ and $\mathbf{V}$ are current injection and bus voltage vectors in the global reference frame. Elements of $\mathbf{I}$ are nonzero for generator and converter buses as loads are represented as constant impedances. Once bus voltages are computed, generator and converter terminal currents (depicted as $I_{t}$ in Fig. 7) are calculated as:

$$
I_{g e n}=I_{Q}+j I_{D}=\frac{E^{\prime \prime}-V_{g t}}{r_{a}+j x_{d}^{\prime \prime}} \quad I_{c o n v}=\frac{E_{v}-V_{p c c}}{Z_{M M C}}
$$

The currents in (26) are in the global reference frame and need to be transformed into relevant local reference frames before being inserted into (25).

\section{Interfacing the different $D A E$}

All the DAE equations for the different test system components presented in the previous sections are interfaced with each other according to Fig. 8. The figure shows interconnections for one of the grids only, but the second system will have a similar interfacing structure. In addition, the DAE equations each block represent are shown in the figure.

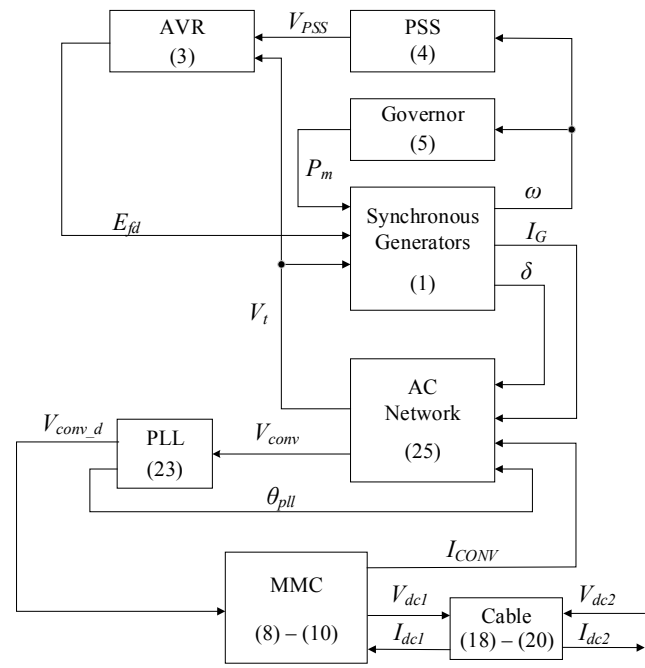

Fig. 8. Complete dynamic model for the test grid

\section{E. Non-Linear Model Simulation}

The non-linear model developed in MATLAB/Simulink was tested for a step change in active power reference. MMC\#1 is operating in dc voltage control, setting the dc link voltage equal to $1 \mathrm{pu}$, while MMC\#2 is operating in power control mode importing 0.2 pu active power into Grid $\mathrm{B}$. The reactive power reference for both converters is set to zero. At $\mathrm{t}=200 \mathrm{~s}$, the reference power was changed by $0.2 \mathrm{pu}$, meaning more power starts to flow into Grid B. This causes an active power imbalance in both grids. Fig. 9 shows the frequencies in both grid after the disturbance.

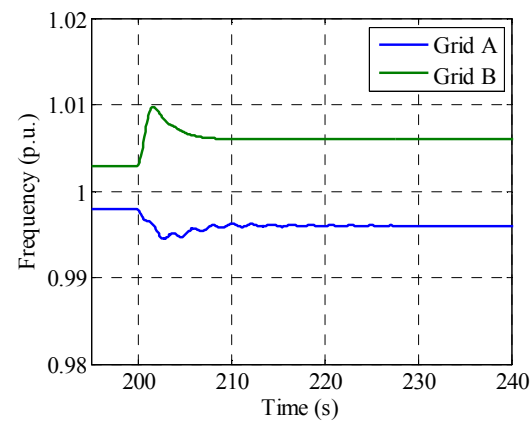

Fig. 9. Frequencies in Grid A and B after step change in power reference of $\mathrm{MMC} \# 2$

The frequency in Grid A drops as there is more load demand in the system (due to the increased power demand through the HVDC from Grid B) than generation, and viceversa for Grid B. The governors in both grids regulate the system frequency by changing generator power output according to their droop setting. The steady-state frequency deviations can be removed by secondary controllers, which are not modelled in this study. 

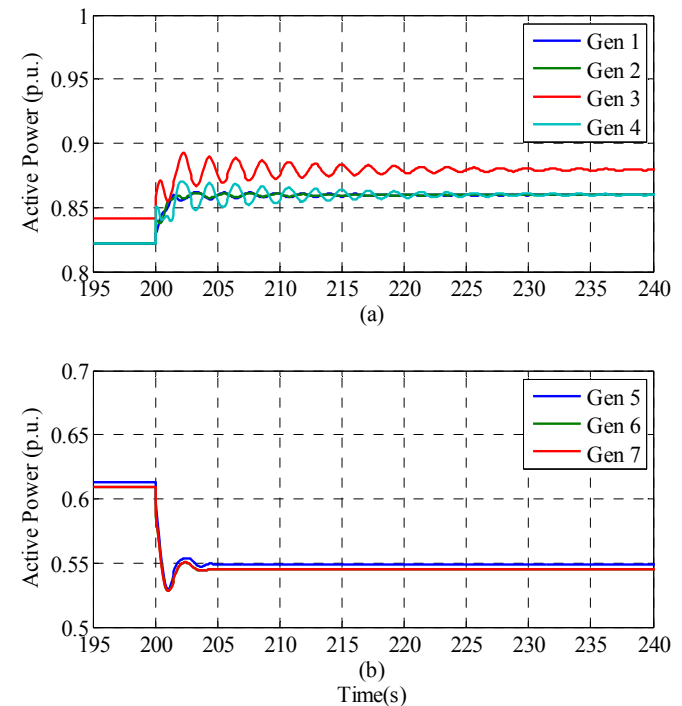

Fig. 10. Active power output of all generators

Fig. 10 shows active power outputs of generators in both grids. There is power system oscillation in Grid A that is clearly visible in the time domain plots of frequency and power output in Fig. 9 and Fig. 10(b). This is studied further with linear analysis.

The effect of step change in power reference can be observed in the internal variables of the converters, in Fig. 11. More precisely, Fig. 11(a) shows the voltage between the dc terminals of MMC\#1, which is being regulated by means of its active current. Furthermore, Fig. 11(b) depicts the zerosequence of the circulating currents of both MMC converters. Note that this variable is strongly related to the dc current flowing out of the converter into the cable, carrying information on the active power transfer in both converters. In this case, the circulating current of $\mathrm{MMC \# 2}$ is stepping up following the reference step change of the active power, whereas the MMC\#1 is counteracting its effect such that its energy sum variable stays regulated to the desired reference, as illustrated in Fig. 11(c).
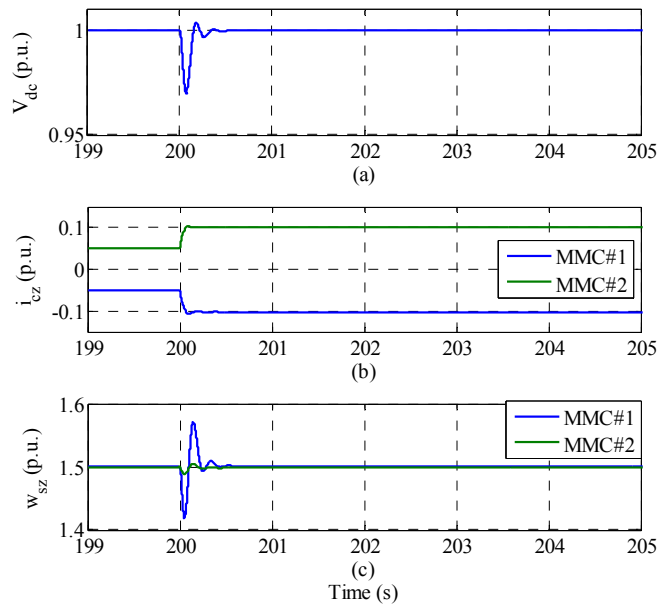

Fig. 11. (a) DC link voltage, (b) Zero sequence circulating current, and (c) zero sequence energy sum

\section{LINEAR ANALYSIS}

\section{A. Linearization}

The small signal model of the hybrid ac/dc system is developed by linearizing the differential and algebraic equations (DAE) presented in the previous sections in MATLAB/Simulink. The state-space representation of the linear system has the form as in (27) where $\mathbf{A}$ is the statematrix, $\mathbf{B}$ is input matrix, $\mathbf{x}$ is a state variable vector and $\mathbf{B}$ is an input vector.

$$
\Delta \dot{\mathbf{x}}=\mathbf{A} \cdot \Delta \mathbf{x}+\mathbf{B} \cdot \Delta \mathbf{u}
$$

The vector of state variables and inputs for synchronous generators with their controllers are:

$$
\begin{aligned}
\mathbf{x}_{\text {gen }} & =\left[\begin{array}{llllllll}
\delta_{i} & \Delta \omega_{i} & E_{q i}^{\prime} & E_{d i}^{\prime} & E_{q i}^{\prime \prime} & E_{d i}^{\prime \prime} & x_{A V R i} & E_{f d i} \\
& \ldots & x_{P S S 1 i} & x_{P S S 2 i} & x_{P S S 3 i} & x_{G O V 1 i} & x_{G O V 2 i}
\end{array}\right]^{T} \\
\mathbf{u}_{\text {gen }} & =\left[\begin{array}{lll}
V_{\text {trefi }} & \omega_{\text {refi }}
\end{array}\right]^{T}
\end{aligned}
$$

Furthermore, the states $\mathbf{x}$ and inputs $\mathbf{u}$ vectors for the MMC converter and controllers are given as:

$$
\begin{aligned}
\mathbf{x}_{M M C} & =\left[\begin{array}{llllllllllll}
i_{c o n v, d} & i_{c o n v, q} & \gamma_{d} & \gamma_{q} & v_{d c} & \rho & i_{c, z} & \xi_{z} & w_{\Sigma} & \kappa_{\Sigma} & \theta_{p l l} & x_{p l l}
\end{array}\right]^{T} \\
\mathbf{u}_{M M C} & =\left[\begin{array}{llllllll}
q_{a c}^{*} & \left|v_{p c c}\right| & \omega_{g} & i_{L} & p_{a c}^{*} & v_{d c}^{*} & w_{\Sigma}^{*}
\end{array}\right]^{T}
\end{aligned}
$$

The MMC adds four extras states compared to a conventional two level VSC converter in a similar configuration [3]. These are $\kappa_{\Sigma}$ and $\xi_{z}$; i.e., the integrator states of the PI-controllers for the aggregated sum energy $w_{\Sigma}$ and equivalent zero-sequence circulating current $i_{c z}$.

The cable has 31 states in total. There are 5 branches on each series section with the inductor dynamics on each branch having one state. In addition to the parallel branches of each pi section, there are the capacitor dynamics, adding 6 states and making the total cables states 31 .

$$
\mathbf{x}_{c a b l e}=\left[\begin{array}{lllllllll}
i_{d c 1_{-} 1} & i_{d c 1_{-} 2} & \ldots & i_{d c 1_{-} 5} & i_{d c 2_{-} 1} & \ldots & v_{d c 1} & \ldots & v_{d c 6}
\end{array}\right]^{T}
$$

\section{B. Modal Analysis}

To understand the effect of HVDC link on the ac grid dynamics, first a modal analysis is carried out for each ac grid. Then the combined hybrid ac/dc system is analyzed.

Grid A is represented by 50 modes in total. It has 4 generators with 6 states variables each, 4 AVRs with 3 state variables, 4 governors with 3 state variables and 1 PSS with 3 state variables. The rotor angle of the reference machine is a redundant state variable. TABLE I. lists modes in Grid A with damping ratio less than $15 \%$. The dominant state variables of each modes are found from participation factor analysis and are also shown. From the dominant state variables for each mode and from the frequency of the modes, it is clear than the least damped modes in Grid A are local and interarea electromechanical modes. 

STATE VARIABLES

\begin{tabular}{|l|c|c|c|}
\hline & Eigenvalues & $\zeta[\%]$ & $\begin{array}{c}\text { Dominant state } \\
\text { variables }\end{array}$ \\
\hline$\lambda_{\mathrm{A} 1,2}$ & $-0.16 \pm \mathrm{j} 3.47$ & 4.7 & $\omega_{3}, \delta_{1}, \delta_{2}, \omega_{4}$ \\
\hline$\lambda_{\mathrm{A} 3,4}$ & $-0.68 \pm \mathrm{j} 6.99 \mathrm{i}$ & 9.7 & $\delta 3, \omega 4, \omega 3$ \\
\hline$\lambda_{\mathrm{A} 5,6}$ & $-0.72 \pm \mathrm{j} 6.81 \mathrm{i}$ & 10.6 & $\delta 2, \omega 2, \omega 1, \delta 1$ \\
\hline
\end{tabular}

TABLE II. shows the least damped modes in Grid B with damping ratio less than $15 \%$. Participation factor analysis shows mode $\lambda_{B 1,2}$ is related to AVR state variables, while both $\lambda_{B 3,4}$ and $\lambda_{B 5,6}$ are mainly related to the speed and angle state variables of the generators in the grid.

TABLE II. POORLY DAMPED MODES IN GRID B

\begin{tabular}{|c|c|c|c|}
\hline & Eigenvalues & $\zeta[\%]$ & Dominant state variables \\
\hline$\lambda_{\mathrm{B} 1,2}$ & $-0.26 \pm \mathrm{j} 4.98$ & 5.31 & $\begin{array}{c}E_{q 7}^{\prime}, E_{f d 7}, E_{q 6}^{\prime}, E_{f d 6}, E^{\prime \prime}{ }_{q 7}, \\
E_{q 6}, \omega_{5}\end{array}$ \\
\hline$\lambda_{\mathrm{B} 3,4}$ & $-1.0 \pm \mathrm{j} 7.49$ & 13.24 & $\omega_{6}, \omega_{7}, \delta_{6}, \delta_{5}$ \\
\hline$\lambda_{\mathrm{B} 5,6}$ & $-1.16 \pm \mathrm{j} 8.26$ & 13.95 & $\omega_{5}, \delta_{5}, \delta_{6}, \omega_{6}, \omega_{7}$ \\
\hline
\end{tabular}

Finally, modal analysis was carried out for the combined hybrid ac/dc system with both Grid A and B linked with MMC-based HVDC. Fig. 12 shows the comparison of modes for Grid A, Grid B and the combined hybrid ac/dc system. Some of the modes in the ac grids are shifted due to coupling by adding the HVDC link.

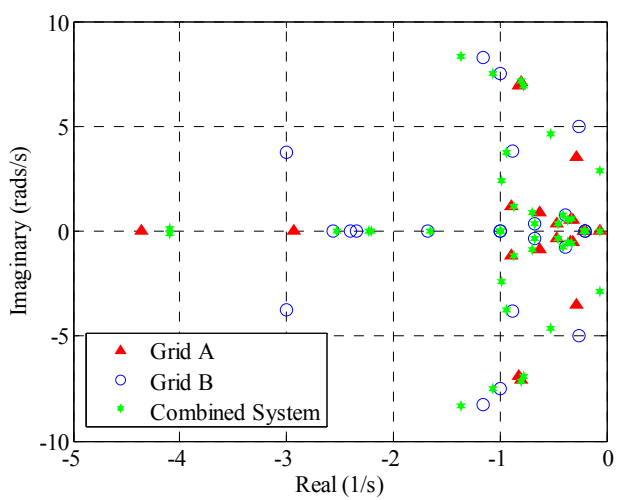

Fig. 12. Comparison of modes for Grid A, Grid B and combined hybrid $\mathrm{ac} / \mathrm{dc}$ test system

TABLE III. lists modes with damping ratio less than $15 \%$ in the combined hybrid ac/dc test system. Most of these modes are local system modes, meaning they are either related to Grid A, Grid B or the dc system. Modes $\lambda_{C S I, 2}$, $\lambda_{C S 9,10,} \lambda_{C S 11,12}$ are Grid A modes related to the generator state variables in the grid. Modes $\lambda_{C S 13,14}$ and $\lambda_{C S 17,18}$ are Grid B modes related to AVR and generator state variables in the grid. Modes $\lambda_{C S 3,4}$ and $\lambda_{C S 5,6}$ are dc cable modes, while modes $\lambda_{C S 15,16}$ and $\lambda_{C S 19,20}$, can be considered interaction modes as the dominant state variables are from the dc cable and the MMC converter control system. Modes with frequencies over $10000 \mathrm{rad} / \mathrm{s}$ are not displayed [18] as they mainly involve high-order cable modes which are largely outside of the upper frequency limit for the cable model used in this work.

The local modes in Grid A, $\lambda_{C S 9,10}$ and $\lambda_{C S 11,12}$, have somewhat shifted to the left in the complex plane with an increased damping ratio of $11.09 \%$ and $11.22 \%$, respectively.. However, the inter-area mode, $\lambda_{A l, 2}$, has become less damped in the combined system $\left(\lambda_{C S I, 2}\right)$.

TABLE III. POORLY DAMPED MODES IN THE COMBINED HYBRID AC/DC TEST SYSTEM

\begin{tabular}{|c|c|c|c|}
\hline & Eigenvalues & $\zeta[\%]$ & $\begin{array}{c}\text { Dominant state } \\
\text { variables }\end{array}$ \\
\hline$\lambda_{\mathrm{CS} 1,2}$ & $-0.065 \pm \mathrm{j} 2.87$ & 2.25 & $\begin{array}{l}\delta_{1}, \omega_{3}, \delta_{2}, \omega_{4}, E_{q 2}^{\prime}, \omega_{1}, \\
E_{q 1}^{\prime}, \theta_{p l l 1}\end{array}$ \\
\hline$\lambda_{\mathrm{CS} 3,4}$ & $-506.2 \pm \mathrm{j} 5867.53$ & 8.60 & $\begin{array}{l}v_{d c 6}, i_{d c 5_{-} 3}, v_{d c 5}, i_{d c 4 \_}, i_{d c 5_{2} 2}, \\
v_{d c 4}, i_{c, z \_}, i_{d c 4 \_}, i_{d c 3_{3}, 3} i_{d c 5 \_}\end{array}$ \\
\hline$\lambda_{\mathrm{CS} 5,6}$ & $-500.72 \pm \mathrm{j} 5107.40$ & 9.76 & $\begin{array}{l}v_{d c 3}, i_{d c 3_{-} 3}, v_{d c 4}, i_{d c 2} 3, v_{d c 6}, \\
v_{d c 2}, i_{d c 3_{-}}, i_{d c s_{-} 3}, i_{d c 2 \_2}, i_{c, z_{3} B}\end{array}$ \\
\hline$\lambda_{\mathrm{CS} 7,8}$ & $-431.64 \pm j 3892.78$ & 11.02 & $\begin{array}{l}v_{d c 4}, i_{d c 2} 3, v_{d c 2}, i_{d c 4 \_}, v_{d c 6}, \\
v_{d c 3}, v_{d c 5}, i_{c, z_{-} B}, i_{d c 2}, i_{d c c_{-} 2},\end{array}$ \\
\hline$\lambda_{\mathrm{CS} 9,10}$ & $-0.8 \pm j 7.16$ & 11.09 & $\delta_{3}, \omega_{4}, \omega_{3}$ \\
\hline$\lambda_{\mathrm{CS} 11,12}$ & $-0.78 \pm j 6.92$ & 11.22 & $\delta_{2}, \omega_{2}, \omega_{1}, \delta_{1}, E_{d 2}^{\prime}$ \\
\hline$\lambda_{\mathrm{CS} 13,14}$ & $-0.53 \pm j 4.60$ & 11.37 & $\begin{array}{l}E_{q 8}^{\prime}, \quad E_{f d 7}, \quad E_{q 6}^{\prime}, \quad E_{f d 6}, \\
E_{q 7}^{\prime \prime}, E_{q 6}^{\prime \prime}\end{array}$ \\
\hline$\lambda_{\mathrm{CS} 15,16}$ & $-298.90 \pm j 2431.23$ & 12.20 & $\begin{array}{llll}\mathrm{v}_{\mathrm{dc} 2}, & \mathrm{v}_{\mathrm{dc} 5}, & \mathrm{i}_{\mathrm{dc} 3} 3 & i_{c, z_{-} \mathrm{B}}, \\
i_{c, z}, \mathrm{~A} & \mathrm{i}_{\mathrm{dc} 4 \_3}, & \mathrm{v}_{\mathrm{dc} 6}, & \mathrm{v}_{\mathrm{dc} 3}, \\
\mathrm{i}_{\mathrm{dc} 2}, & w_{\Sigma z B} & & \\
\end{array}$ \\
\hline$\lambda_{\mathrm{CS} 17,18}$ & $-1.07 \pm j 7.53$ & 14.05 & $\omega_{7}, \delta_{6}, \omega_{6}, \delta_{5}$ \\
\hline$\lambda_{\mathrm{CS} 19,20}$ & $-181.96 \pm \mathrm{j} 1257.6$ & 14.32 & 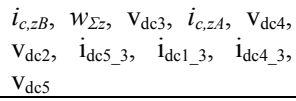 \\
\hline
\end{tabular}

In general, the case study shows that the interconnection of stable subsystems, namely two ac grids and a dc link, influences some of the modes of the combined system. However, due to the conservative tuning of the different elements in each and every subsystem the overall system is also stable.

\section{CONCLUSION}

This paper presented a comprehensive modelling approach to obtain a small-signal model of a Modular Multilevel Converter (MMC)-based High Voltage Direct Current (HVDC) link interfacing two large asynchronous ac systems. Dynamic components of the ac grid, MMC converter and its control system, and the dc cable were modelled in detail. The modal analysis results show that both the individual ac grids and the combined system are stable. Further analysis on modes with damping ratio less than $15 \%$ showed that the modes are related to a single sub-system (either of the ac grids or the dc system). However, some modes have strong participation from both MMC and cable state variables.

The combined modeling approach presented in this paper can be used to study the effect of faster ac system 
phenomena such as inertial response delivered through the HVDC link.

\section{ACKNOWLEDGMENT}

The research of Jef Beerten is funded by a postdoctoral fellowship of the Research Foundation - Flanders (FWO).

\section{REFERENCES}

[1] S. Akkari, J. Dai, M. Petit, P. Rault, and X. Guillaud, "Small-Signal Modelling for in-Depth Modal Analysis of an MTDC System," in Electrical Power and Energy Conference (EPEC), 2015 IEEE, 2015, pp. 233-238.

[2] J. Beerten, G. Bergna-Diaz, S. D' Arco, and J. A. Suul, "Comparison of Small-Signal Dynamics in Mmc and Two-Level VSC HVDC Transmission Schemes," in ENERGYCON, ed. Leuven, Belgium, 2016.

[3] G. Bergna-Diaz, J. A. Suul, and S. D. Arco, "Small-Signal State-Space Modeling of Modular Multilevel Converters for System Stability Analysis," in 2015 IEEE Energy Conversion Congress and Exposition (ECCE), 2015, pp. 5822-5829.

[4] M. K. Zadeh, M. Amin, J. A. Suul, M. Molinas, and O. B. Fosso, "Small-Signal Stability Study of the Cigre DC Grid Test System with Analysis of Participation Factors and Parameter Sensitivity of Oscillatory Modes," in Proc. Power Systems Computation Conference (PSCC), Wroclaw, Poland, 2014

[5] O. Kotb, M. Ghandhari, R. Eriksson, and V. K. Sood, "On Small Signal Stability of an AC/DC Power System with a Hybrid MTDC Network," Electric Power Systems Research, vol. 136, pp. 79-88, 2016.

[6] N. R. Chaudhuri, R. Majumder, B. Chaudhuri, and P. Jiuping, "Stability Analysis of VSC MTDC Grids Connected to Multimachine AC Systems," IEEE Transactions on Power Delivery, vol. 26, pp. 2774-2784, 2011.

[7] P. Rault, X. Guillaud, F. Colas, and S. Nguefeu, "Investigation on Interactions between AC and DC Grids," in Proc. IEEE PowerTech, Grenoble, France, 2013.
[8] A. Lesnicar and R. Marquardt, "An Innovative Modular Multilevel Converter Topology Suitable for a Wide Power Range," in Power Tech Conference Proceedings, 2003 IEEE Bologna, 2003, p. 6 pp. Vol.3.

[9] R. Adapa, "High-Wire Act: Hvdc Technology: The State of the Art," IEEE Power and Energy Magazine, vol. 10, pp. 18-29, 2012.

[10] S. D. Arco, J. A. Suul, and M. Molinas, "Implementation and Analysis of a Control Scheme for Damping of Oscillations in VSCBased HVDC Grids," in Power Electronics and Motion Control Conference and Exposition (PEMC), 2014 16th International, 2014, pp. 586-593.

[11] J. Freytes, L. Papangelis, H. Saad, P. Rault, T. Van Cutsen, and X. Guillaud, "On the Modeling of Mmc for Use in Large Scale Dynamic Simulations," Proceedings of the 2016 IEEE Power Systems Computation Conference PSCC, Genoa, Italy, 20th - 24th June 2016. 2016.

[12] A. Jamshidifar and D. Jovcic, "Small-Signal Dynamic DQ Model of Modular Multilevel Converter for System Studies," IEEE Transactions on Power Delivery, vol. 31, pp. 191-199, 2016.

[13] P. Kundur, Power System Stability and Control: McGraw-Hill Inc., 1994.

[14] P. M. Anderson and A. A. Fouad, Power System Control and Stability: IEEE Press, 1993.

[15] J. Machowski, J. Bialek, and J. Bumby, Power System Dynamics: Stability and Control: WILEY, 2008.

[16] A. Antonopoulos, L. Angquist, and H. P. Nee, "On Dynamics and Voltage Control of the Modular Multilevel Converter," in Power Electronics and Applications, 2009. EPE '09. 13th European Conference on, 2009, pp. 1-10.

[17] L. Harnefors, A. Antonopoulos, S. Norrga, L. Angquist, and H. P. Nee, "Dynamic Analysis of Modular Multilevel Converters," IEEE Transactions on Industrial Electronics, vol. 60, pp. 2526-2537, 2013.

[18] J. Beerten, S. D' Arco, and J. A. Suul, "Frequency-Dependent Cable Modelling for Small-Signal Stability Analysis of VSC-HVDC Systems," IET Generation, Transmission \& Distribution, vol. 10, pp. 1370-1381, 2016 